\title{
Multi-interface Level Sensors and New Development in Monitoring and Control of Oil Separators
}

\section{Syed Faisal Ahmed Bukhari ${ }^{*}$ and Wuqiang Yang}

School of Electrical and Electronic Engineering, The University of Manchester, P.O. Box 88, Manchester M60 1QD, United Kingdom

* Author to whom correspondence should be addressed. Email: s.bukhari@postgrad.manchester.ac.uk Received: 26 August 2005 / Accepted: 24 February 2006 / Published: 7 April 2006

\begin{abstract}
In the oil industry, huge saving may be made if suitable multi-interface level measurement systems are employed for effectively monitoring crude oil separators and efficient control of their operation. A number of techniques, e.g. externally mounted displacers, differential pressure transmitters and capacitance rod devices, have been developed to measure the separation process with gas, oil, water and other components. Because of the unavailability of suitable multi-interface level measurement systems, oil separators are currently operated by the trial-and-error approach. In this paper some conventional techniques, which have been used for level measurement in industry, and new development are discussed.
\end{abstract}

Keywords: Level sensor, multi-interface measurement, electrical capacitance tomography (ECT). 


\section{Introduction}

Measurement of liquid level in storage and processing vessels, tanks, wells, reservoirs and hoppers is commonly needed. There is a great diversity of situations, which require level measurement, ranging from pure water to viscous, sticky and corrosive fluids. The environment for level sensors varies from vacuum to high pressure and from below zero Celsius degree to high temperature. Because of the involvement of different situations, many types of level sensors have been developed.

Nowadays, the control strategies in use with crude oil separators are simple due to the difficulties in finding suitable multi-interface level measurement systems. Accurate measurement of multi-interface levels in crude oil separators is regarded as a challenge for hydrocarbon processing. A number of different methods have been developed, e.g. externally mounted displacers, differential pressure transmitters, ultrasonic transducers, single-electrode and multi-electrode capacitance sensors. The methods used in the oil industry for measuring the interfaces in crude oil separators are limited because of the request on high reliability, variety of fluids, harsh environments and intrinsic safety issues. It is particularly important for the offshore oil companies to deal with this problem because crude oil from undersea contains a huge amount of water, which should be separated on site to avoid the additional transportation cost. Therefore, it is necessary to investigate suitable sensors for an accurate and reliable measurement of different interface levels.

A crude oil separator (see Figure 1) normally contains gas, oil, water, emulsion and sludge. Due to the limited space in an offshore platform, effective monitoring and efficient control of crude oil separators would result in huge savings to the oil companies and reduce environmental pollution. Some other applications of multi-interface level measurement systems are petrol/kerosene storage tanks in petrol stations and fuel storage tanks in airports. For these applications an ideal multi-interface level measurement system should have the following characteristics:

(1) Accurately locating interfaces between different materials, e.g. gas-oil, oil-emulsion and emulsion-water,

(2) Low maintenance cost,

(3) Self-cleaning,

(4) Intrinsically safe.

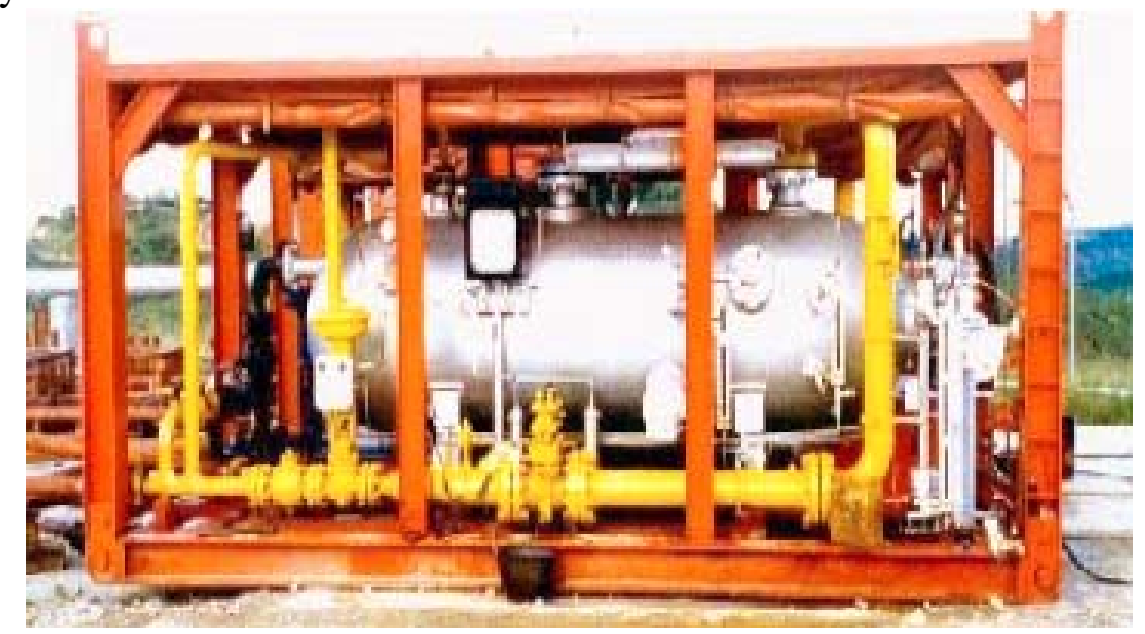

Figure 1. Conventional oil separator. 


\section{Level measurement techniques}

The operation of an oil separator relies on the measurement of interfaces between different materials. If a conventional capacitance level sensor with only a single-electrode is used, two main problems are encountered: (1) Temperature compensation is required because the capacitance measurement is greatly affected by temperature, and (2) it is unable to measure more than one interface. Research has been carried out to investigate multi-interface level measurement systems.

\section{Externally mounted displacers}

Externally mounted displacers are commonly used for the interface level measurement [1]. This technique makes use of the difference in densities between two types of liquid being measured, e.g. oil and water. A displacer used for oil/water interface measurement must have a density between the densities of oil and water.

As the interface moves up and down in a separator, the position of displacer is mechanically transferred to a read-out system (see Figure 2). Using two different displacers with different densities, both the oil/gas and water/oil interfaces can be measured. However, the presence of an emulsion layer will disturb the measurement and also the foam thickness cannot be measured by this technique.

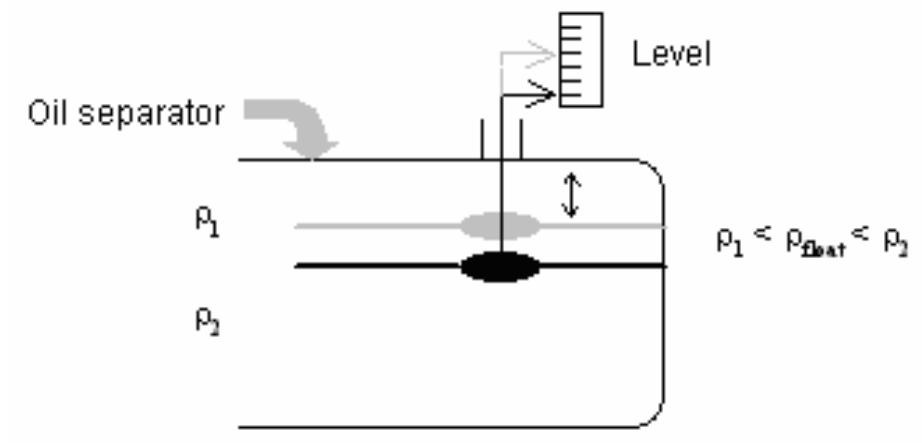

Figure 2. Level monitoring by displacer.

Differential pressure transmitter

Various interfaces in an oil separator may be detected using differential pressure transmitters (see Figure 3).

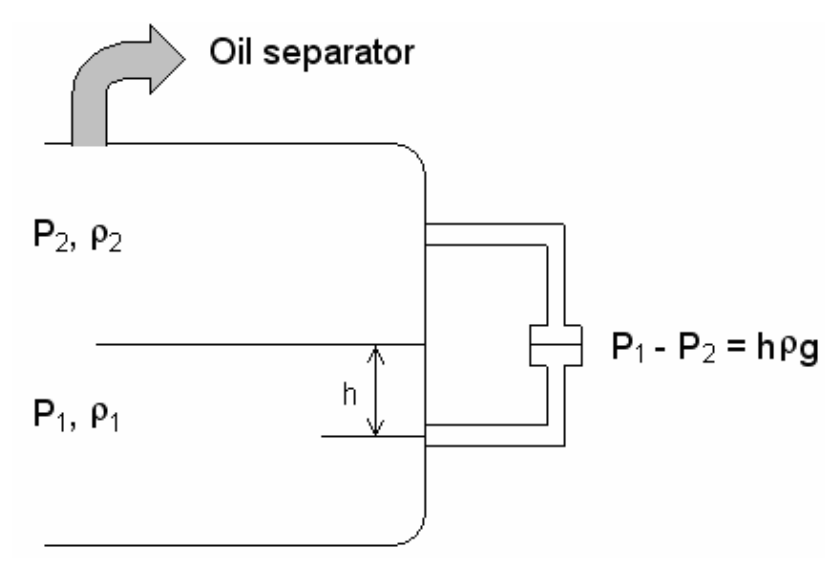

Figure 3. Differential pressure based level detection. 
In this technique differential pressure is measured between the oil and water phases and between the oil and air phases. The densities of different phases are used to calculate the heights of oil and water. The disadvantage of this technique is that emulsion and foam cannot be measured. The other problems with this method is that the pipe work connecting differential pressure transmitters to different phases inside separators may get blocked due to sand and wax formation. Also, heating and thermal insulation are required.

\section{Ultrasonic transducers}

An ultrasonic level measurement system consists of an ultrasonic transmitter and an ultrasonic receiver (see Figure 4). The reflected signals from different interface levels, in the measurement volume, are received by the ultrasonic receiver, as the signals are the function of the density of the medium and the speed of sound in the medium. Different interfaces in a separator can be obtained by signal processing. This method has been used for continuous and discrete level monitoring [1, 2].

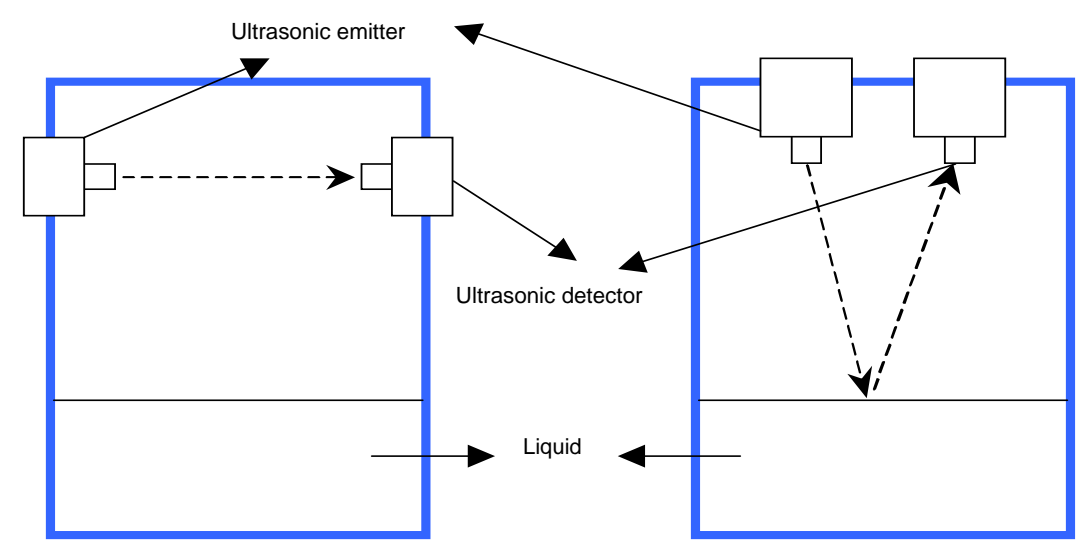

Figure 4. Ultrasonic transducers.

At the Christian Michelson Research (CMR) in Norway, a clamp-on sensor has been developed for liquid level measurement, which is called Ultrasonic Interface Level Detector (UID). Figure 5 shows the UID system with a separator. In this technique the ultrasonic transducer is mounted on the outside of the bottom wall of an oil separator. It contacts the separator directly, and transmits and receives ultrasonic pulses along the vertical axis.

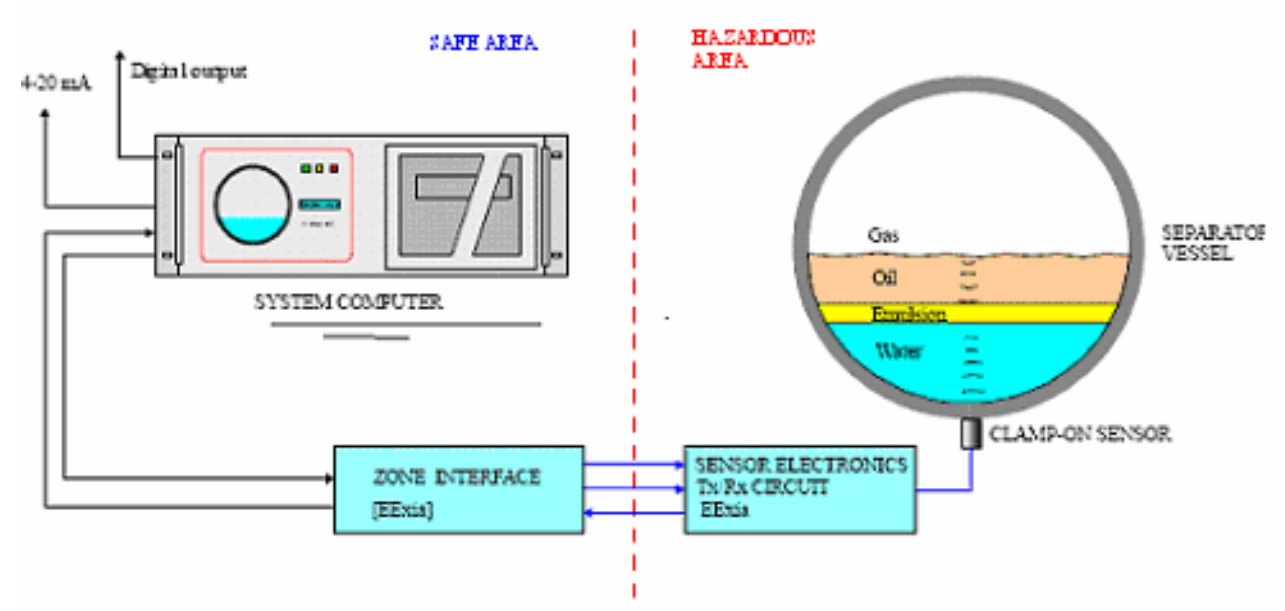

Figure 5. Ultrasonic interface level detector with a separator vessel. 
The measurement is based on pulse echo [3] and the levels are estimated by combining the measured time-of-flight of echoes from the fluid interfaces with the speed of sound in the propagation media. The advantages of this system are that it is non-intrusive and easy to install on existing and new separators, and no calibration is needed. This system is in commercial use by Esso and Statoil. The disadvantage of this system is that it has difficulty in monitoring emulsion and foam [4]. Also, the presence of air bubbles in the liquid attenuates/scatters the ultrasound waves. It becomes a big problem when a significant amount of air is present in the liquid. The presence of sand also causes problems in the operation of UID.

\section{Gamma ray sensors}

In this method, low energy gamma rays are used. A vertical array of low energy gamma-ray sources emits radiation, which is monitored by two vertical arrays of Geiger detectors. The material present in a separator attenuates the radiation "seen" by the detector. The amount of attenuation is determined by the density of the medium and readings are collected by each of the detectors in the vertical assembly. The collected data are then converted to the density measurement.

The Geiger detectors can be operated in two modes, current mode and pulse mode. The current mode is used to quantify the intensity of a gamma radiation field and the pulse mode to qualify or analyse the type and energy of radiation. This system is easy to install. Since it has no moving parts, essentially no maintenance is required $[5,6]$. The Geiger detectors are rugged and have a design life of 15 years. This system is useful in measuring the vertical distribution of the various phases in a vessel. Also, it gives information regarding inter-phase mixing. The disadvantage with the nucleonic type interface level sensor is the presence of hazardous nucleonic radiation.

\section{Multi-electrode capacitance level sensors}

To overcome the two problems with the single-electrode measurement technique mentioned in the beginning of section before, multi-electrode capacitance level sensors (see Figure 6) have been developed at the UMIST (now the University of Manchester) [7]. In this system, a capacitance sensor with 64 segmented electrodes enables the interfaces to be located based on differences in dielectric constants of materials. A normalised difference algorithm is incorporated to obtain the interfaces of gas/oil and oil/water using the data obtained from capacitance values.

$$
y(i)=\frac{x(i+1)-x(i)}{x(i)} \text { with } \mathrm{i}=1,2,3, \ldots, 63
$$

where, $y(i)$ is the normalised difference and $x(i)$ is the capacitance reading.

If two electrodes corresponding to $\mathrm{x}(\mathrm{i})$ and $\mathrm{x}(\mathrm{i}+1)$ are immersed in the same material, $\mathrm{y}(\mathrm{i})$ is small. Otherwise, y(i) is large. In this method sharp interfaces can be easily identified. Foam is distinguished from other materials by a signal spectrum obtained by FFT analysis. One problem associated with this method is that if a detection electrode is merged in a highly conductive liquid, such as saline water, short circuit to the electrode would happen because of the high conductivity. 


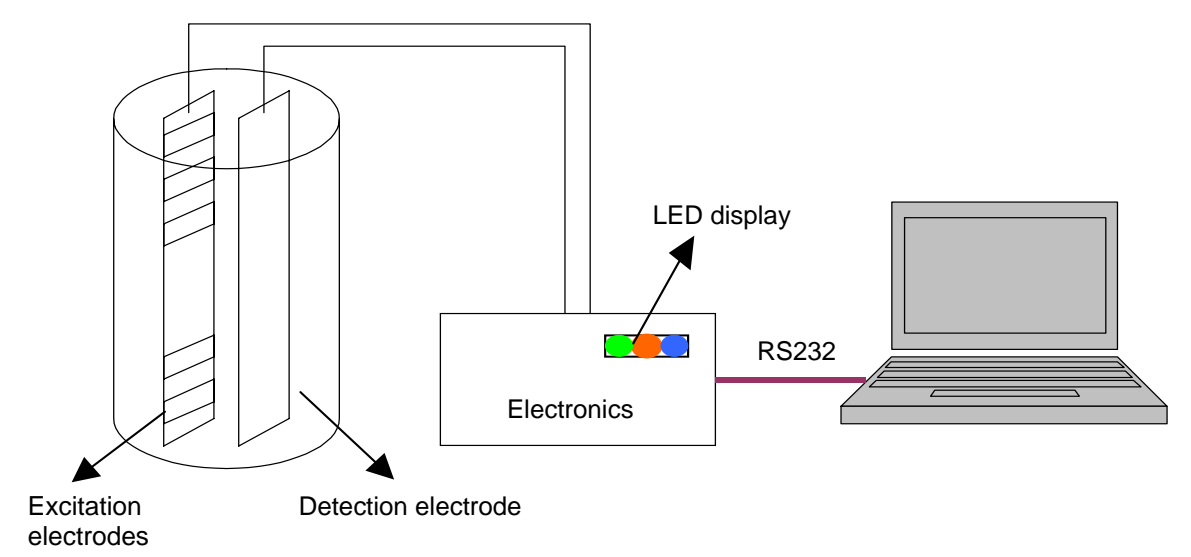

Figure 6. Multi-electrode capacitance level system.

Shell has developed the first capacitance-based instrumentation for multi-interface level measurement [8]. Further work using segmented capacitance electrodes has been done at UMIST to deal with the saline water [2]. In this work, twelve excitation electrodes and one detection electrode were used. For capacitance measurement, a charge/discharge circuit was adopted because it is stray immune and capable of operating at high frequencies to reduce the effects of the liquid conductivity on the measurements. Also, the charge/discharge circuit has advantages of simplicity and low cost [9]. Another effort was made on designing a capacitance transducer based on the charge/discharge principle [10]. In this approach, the presence of foam is obtained by processing the fluctuating component of the capacitance signal, which reflects the inherently unstable feature of the foam, whereas the multi-interface levels are reconstructed by processing the steady-state component of the measured capacitances.

\section{Other techniques}

The other techniques in use for interface level measurement are based on inductive and thermal measurements. A number of different approaches, direct and indirect, have been adopted for the measurement of inductance. In the direct approach, electrodes are placed in the medium being measured. In the indirect approach, an alternating magnetic field is applied to induce a current in the medium as a function of the conductivity of the medium. The direct approach is not viable due to the possible building-up of various insulating layers in a separator. The indirect approach is also not feasible due to its ability for water detection only. Instead, capacitance measurement is needed for identifying low conductivity materials, like oil and gas.

Thermal measurement involves measuring of different thermo-dynamic properties of the liquid surrounding a thermal sensor. This type of sensor is intrusively present in a separator. The inner part of this system, which consists of a rod, should be kept at a constant temperature by continuously circulating a liquid with temperature control. The disadvantages of this technique are intrusiveness and involvement of the continuously circulating liquid.

Hall-effect sensors have also been identified and investigated at the University of Manchester for this application. An extremely sensitive measuring circuit for the sensors has been developed [11]. This circuit can be used to develop a multi-interface sensor comprising of capacitance and Hall-effect 
sensing elements. The preliminary results obtained in the laboratory are extremely promising. Table 1. summarizes different multi-interface level measurement techniques.

Table 1. Comparison of multi-interface level measurement techniques.

\begin{tabular}{|c|c|c|c|}
\hline S. No. & $\begin{array}{l}\text { Level measurement } \\
\text { techniques }\end{array}$ & Advantages & Disadvantages \\
\hline 1. & Externally mounted displacers & - Simple to implement. & $\begin{array}{l}\text { - Emulsion layer cannot be } \\
\text { detected. }\end{array}$ \\
\hline 2. & Differential pressure transmitters & $\begin{array}{l}\text { The densities of different } \\
\text { phases are used for } \\
\text { calculation. }\end{array}$ & $\begin{array}{l}\text { - Intrusive technique. } \\
\text { - Heating and thermal } \\
\text { insulations are required. }\end{array}$ \\
\hline 3. & Ultrasonic transducers & $\begin{array}{l}\text { - This can be used for } \\
\text { continuous and discrete } \\
\text { level monitoring. } \\
\text { - It is non-intrusive and easy } \\
\text { to install on existing and } \\
\text { new separators. } \\
\text { - } \quad \text { No calibration is needed. }\end{array}$ & $\begin{array}{l}\text { - It has difficulty in } \\
\text { monitoring emulsion and } \\
\text { foam. } \\
\text { - Presence of air bubbles } \\
\text { in the liquid attenuates } \\
\text { the ultrasound waves. }\end{array}$ \\
\hline 5. & Gamma ray sensors & $\begin{array}{l}\text { - Useful in measuring the } \\
\text { vertical distribution. }\end{array}$ & $\begin{array}{l}\text { Presence of hazardous } \\
\text { nucleonic radiation. }\end{array}$ \\
\hline 6. & $\begin{array}{l}\text { Multi-electrode capacitance } \\
\text { sensors }\end{array}$ & $\begin{array}{ll}- & \text { Different interface } \\
& \text { levels can be detected. }\end{array}$ & $\begin{array}{l}\text { - Conductive liquids cause } \\
\text { problems. }\end{array}$ \\
\hline 7. & Inductive measurement & $\begin{array}{l}\text { - Problem due to } \\
\text { conductive liquid can be } \\
\text { avoided. }\end{array}$ & $\begin{array}{l}\text { - Building up of various } \\
\text { insulating layers in a } \\
\text { separator. }\end{array}$ \\
\hline 8. & Thermal measurement & $\begin{array}{l}\text { - Can be used effectively } \\
\text { for level detection. }\end{array}$ & $\begin{array}{l}\text { - Continuous circulating } \\
\text { liquid is required. }\end{array}$ \\
\hline
\end{tabular}

\section{Novel approach for oil separator monitoring and control}

In this work, a novel approach has been used for oil separator visualisation. If conventional ECT calibration method, i.e. to use only high and low permittivity materials for sensor's calibration, is applied then air and oil cannot be imaged in different colours. This is because the permittivity values of air and oil are close, i.e. 1 and 2.1, respectively. Water, however, has a permittivity value of 80. To image air, oil and water, an ECT sensor is calibrated by air, oil and water instead of air and water only. When a set of capacitance has been acquired from an ECT sensor surrounding an oil separator, one image is generated using the air-oil calibration data to reflect the contrast between air as one component and oil and water as another component. A second image is then generated using the airwater calibration data to reflect the contrast between air and oil as one component and water as another component. The next step is to use image fusion, i.e. to combine the two images together to illustrate a three-component distribution $[12,13]$. This is shown in Figure 7.

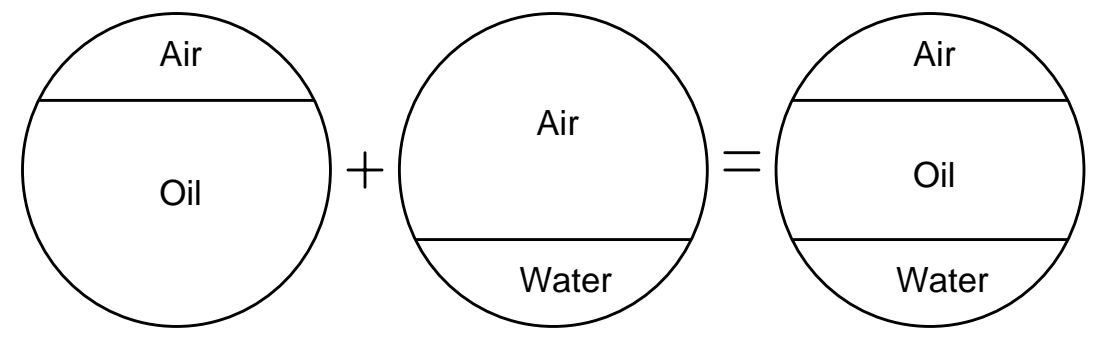

Figure 7. Image fusion to show three-component distribution. 
Recently, an oil separator test rig (Figure 8) has been developed at the University of Manchester [14]. It includes a high sensitivity electrical capacitance tomography (ECT) system based on a commercial impedance analyser (HP4192A), which has 10 times higher sensitivity than conventional ECT systems [10]. Other main units in this system are a multiplexer box, an IEEE-488 card and a host computer. The impedance analyser is designed to measure a wide range of impedance, phase, delay and other parameters. The multiplexer box is designed for the data acquisition system to measure the impedance between the sensor electrodes and to ensure the minimum stray capacitance. This is accomplished with reed relay switches, which can be set to connect any terminal to the excitation or detection terminal of the impedance analyser. A total of 16 electrodes can be independently controlled for 4 different operation modes, i.e. excitation, detection, floating and ground. The IEEE-488 card is employed to make an interface between the host computer and the impedance analyser. The computer is used to control the operation of both the impedance analyser and the multiplexer, and to co-ordinate the measurement procedure. It is also used for processing the data to reconstruct an image corresponding to the material distribution inside the sensor. Figure 9 shows the main parts of the impedance analyser based ECT system.

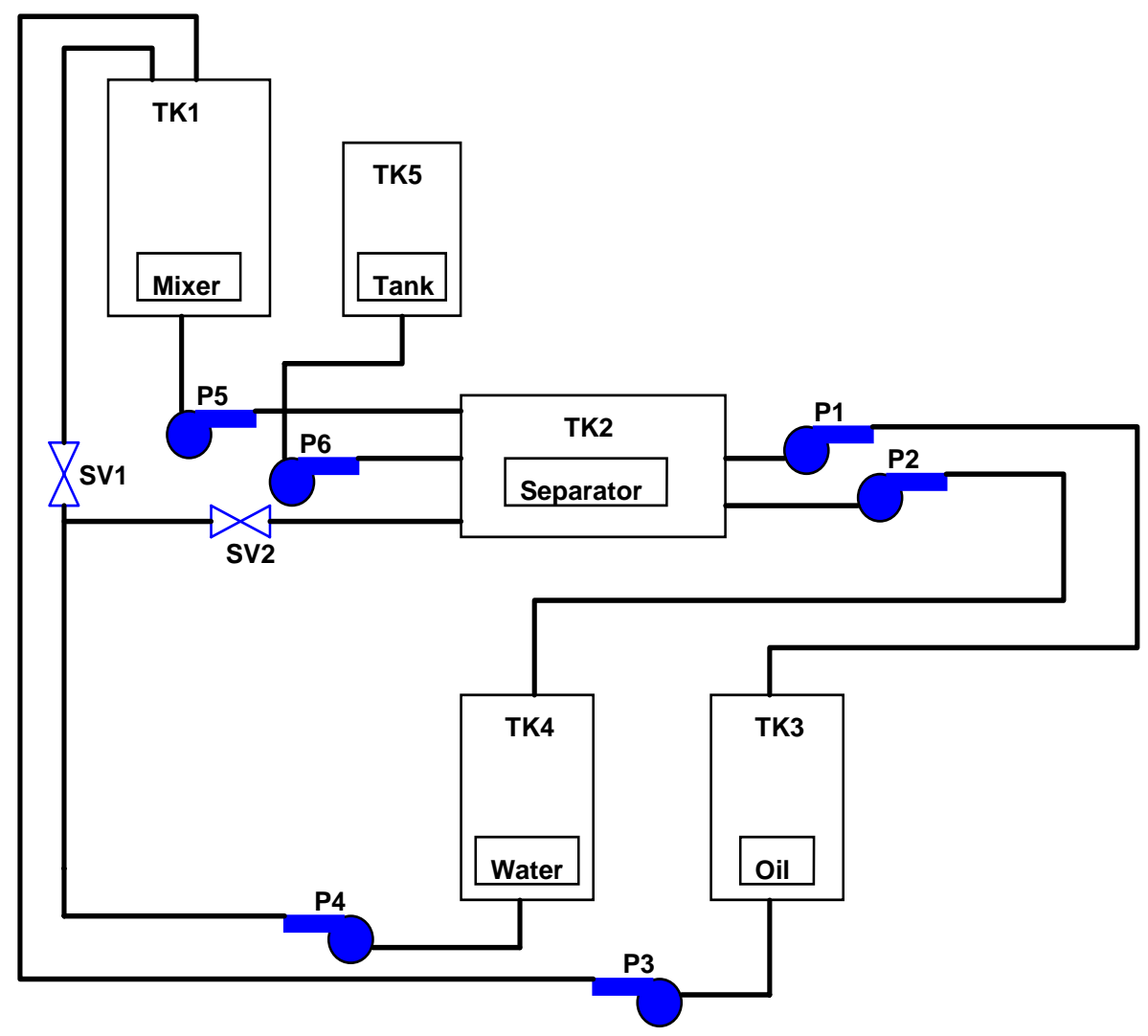

Figure 8. Test rig flow diagram.

As control strategy, a knowledge-based technique has been implemented. A large number of images for different interface levels of oil, water and air have been obtained and processed using image fusion technique discussed above. These images form the knowledge base. Then, a hybrid technique based on principle component analysis (PCA) and cluster analysis has been considered for decision making. The PCA is used for generating precise prior information using the model-based approach and then, cluster analysis is used to see the closest stored condition of the current situation, i.e. the measured image, thus taking advantages of both techniques. This control strategy has been tested using the experimental 
test rig and promising results have been obtained. This hybrid approach has been used in the past only for the analysis of water column distributions in the field of oceanography [16]. It has not been used in the oil industry for oil separator control. The resulting control system is an adaptive control system as if the control action for the current situation is not available in the knowledge base, then using Euclidean distance the action for the closest data set is taken [12].

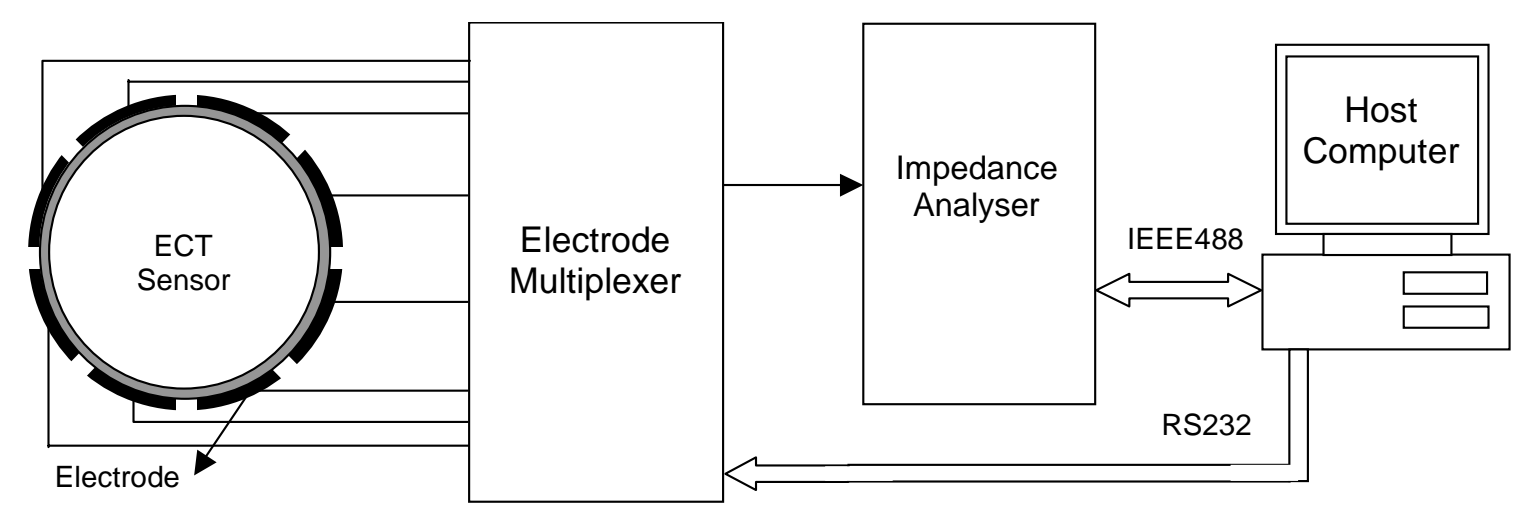

Figure 9. Impedance analyser-based ECT system.

\section{Conclusions}

The oil industry can benefit from employing suitable multi-interface level measurement systems in oil separators. However, the in-use control strategies for oil separators are simple due to the lack of such measuring systems. The use of suitable multi-interface level measurement systems would result in efficient separators, compact separator design, less chemical use and good quality products. To achieve this, different techniques have been investigated, e.g. externally mounted displacers, differential pressure transmitters, ultrasonic transducers, gamma sensors, capacitance sensors with single or multi-electrodes, inductive and thermal sensors. A few commercial systems are available using ultrasonic and gamma radiation techniques. However, incapability in the presence of air bubbles and hazardous nucleonic radiation make them unpopular in the industry.

An ECT system based on an impedance analyser has been developed. The images obtained from the ECT system have been used for implementing a knowledge-based control system. The experimental results are promising. It is concluded that ECT can be used effectively for oil separator monitoring and control.

\section{Acknowledgements}

Syed Faisal Ahmed Bukhari would like to take this opportunity to thank the Ministry of Education, Pakistan for supporting his PhD study at the University of Manchester. Norman Axon is thanked for his help in constructing the oil separator test rig. Rehan Hafiz, Shahram Mohanna and Uzair Tahir are thanked for their valuable discussions. 


\section{References}

1. Hjertaker, B.T.; Johansen, G.A.; Jackson, P. Recent developments in hydrocarbon separator interface imaging, Proc. of SPIE, 2001, 4188, 81.

2. Akhneifer, A.E.M. Development of a multi-interface level measurement system, MSc dissertation, Department of Electrical Engineering and Electronics, UMIST, Manchester, UK, 1999.

3. Bjelland, C. Ultrasonic interface level detection-UID, http://www.cmr.no, 2005.

4. Beck, M.S.; Waterfall, R.C.; Williams, R.A.; Yang, W.Q.; Chen, Y.W.; Banner, A.; Brant, M.R. Interface measurements in three-phase separators, Final report of project PI5 "Design and instrumentation of primary separation systems, Phase I”, 1994, Process Tomography Group, UMIST.

5. Darwood, M.; James, K.; Jackson, P.; Hewitt, P. Density profiling in multiphase systems using Gamma ray absorption, Proc. of $3^{\text {rd }}$ World Congress on Industrial Process Tomography, 2-5 Sept. 2003, Banff, Canada, pp. 870.

6. Lees, R.P. Increase oil production and reduce chemical usage through separator level measurement by density profiling, 2005, http://www.isa.org/journals/intech/ETCON2046.pdf

7. Yang, W.Q.; Brant, M.R.; Beck, M.S. A multi-interface level measurement system using a segmented capacitance sensor for oil separators, Meas. Sci. Technol., 1994, 5(9), 1177.

8. Shell International Research, Level Gauge, European Patent, 1984, Application No. 84201889.7.

9. Yang, W.Q. Hardware design for electrical capacitance tomography systems, Meas. Sci. Technol., 1996, 7, 225.

10. Shi, T.M.; Xie, C.G.; Huang, S.M.; Williams, R.A.; Beck, M.S. Capacitance-based instrumentation for multi-interface level measurement, Meas. Sci. Technol., 1991, 2, 923.

11. Syed, T. 2D/3D magnetic arrays for imaging, MSc dissertation, Department of Electrical Engineering and Electronics, UMIST, Manchester, UK, 2004.

12. Bukhari, S.F.A.; Ismail, I.; Yang, W.Q. Visualising oil separator vessel and decision-making for control, 4th World Congress on Industrial Process Tomography, Aizu, Japan, 5-8 September, 2005, pp. 855-860.

13. Yang, W.Q. Tomographic and non-tomographic instrumentation for multiphase separation monitoring and control, Proc. Multiphase Separation and Multiphase Pumping Technologies Conference, Aberdeen, UK, 1-2 September 2005.

14. Bukhari, S.F.A.; Yang, W.Q. Tomographic imaging technique for oil separator control, Proc. $3^{\text {rd }}$ International Symposium on Process Tomography, Lodz, Poland, 9-10 September, 2004, pp. 27-31.

15. Yang, W.Q.; York, T.A. New AC-based capacitance tomography system, IEE Proc. Sci., Meas. and Technology, 1999, 146, 47.

16. Burd, A.B.; Jackson, G.A. An analysis of water column distributions in Florida Bay, Estuaries, 2002, 25(4A), 570.

(C) 2006 by MDPI (http://www.mdpi.org). Reproduction is permitted for non-commercial purposes. 